\title{
Weak Interaction Matrix Elements in Strong Coupling Lattice QCD
}

\author{
Apoorva Patel \\ Theory Division, CERN, Geneva, Switzerland
}

Talk presented at the International Symposium on Field Theory of the Lattice : LAT87 Seillac, France, Sept.28-Oct.2, 1987

\begin{abstract}
Amplitudes of many non-leptonic processes require a non-perturbative calculation of matrix elements between pseudo-Goldstone boson states, after the standard perturbation theory in the Electro-Weak sector. Several such matrix elements are estimated using the techniques of strong coupling lattice QCD.
\end{abstract}

CERN-TH-4899/87

November 1987 
Though leptonic Electro-Weak processes are well understood in the framework of perturbation theory, that is not the case with non-leptonic processes. The missing component in the latter case is the knowledge of how non-perturbative QCD contributions modify the amplitudes at low energy scales. Some well known examples are :

1) $K \rightarrow 2 \pi$ non-leptonic decays and $\Delta I=1 / 2$ rule.

2) $K^{0}-\bar{K}^{0}$ mixing and the CP violation parameter $\epsilon$.

3) CP violation in $K \rightarrow 2 \pi$ decays and the parameter $\epsilon^{\prime}$.

4) $\pi^{+}-\pi^{0}$ mass difference.

A common feature among the processes listed above is that they involve only the pseudoGoldstone boson fields. In such cases, the pattern of chiral symmetry breaking restricts the form of the various amplitudes, and one hopes to achieve an understanding of the remaining parameters in a theoretical framework.

The strategy in the computation of such amplitudes [1] is to start with the standard $S U(3)_{c} \times S U(2)_{L} \times U(1)_{Y}$ model, and carry out a perturbative expansion in the Electro-Weak interactions. Then the energy scale is gradually lowered evolving the various interactions using renormalisation group equations. When the energy scale becomes comparable to a heavy field mass, the heavy field is integrated out using the Operator Product Expansion, leaving behind a Lagrangian containing effective interactions of the lighter fields. Continuing this process, finally one arrives at a Lagrangian containing renormalised interactions, whose matrix elements are to be evaluated at low energy scales.

$$
L_{\text {int }}=\sum_{i} C_{i}(\mu) O_{i}(\mu)
$$

Here both the effective operators $O_{i}$ and the Wilson coefficients $C_{i}$ are defined using a particular regularisation scheme and they depend on the measurement scale $\mu$. The matrix elements of $L_{\text {int }}$ between hadronic states however have no dependence either on the regularisation or the scale $\mu$. The coefficients $C_{i}$ are generally calculated using weak coupling perturbation theory, while the quantities $\left\langle O_{i}\right\rangle \equiv<h_{f}\left|O_{i}\right| h_{i}>$ are estimated by non-perturbative methods.

The lattice formulation of QCD provides an explicitly non-perturbative framework for evaluating the $\left\langle O_{i}\right\rangle$. In this regularisation, one can imagine lowering the scale $\mu$ all the way down to the strong coupling limit. The aim of this talk is to present the methodology for determining the $\left\langle O_{i}\right\rangle$ in this limit. I use the staggered fermion formulation, which by preserving a subset of the continuum chiral symmetries at all scales produces Goldstone bosons with the correct chiral behaviour.

Several drawbacks of such an analysis are already known :

- One is working at the "wrong" fixed point of the theory. The weak coupling scaling behavior is lost and the hadron spectrum cannot be fit to better than $\sim 30 \%$ accuracy.

- The complete set of continuum chiral symmetries does not exist on the lattice and mixing between different lattice operators takes place.

- Perturbative calculations of $C_{i}$ and operator mixing cannot be trusted. They can only be regarded as a qualitative guide.

These reasons make it clear that the results obtained in the strong coupling limit should be interpreted only as trends and not as quantitative predictions. Nevertheless, there are certain welcome features in pursuing this approach :

- One still has a regulated theory of (partially) conserved currents and their corresponding charges. This has a certain degree of similarity with the chiral Lagrangian description 


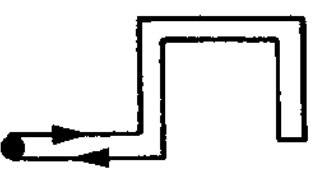

$O(N)$

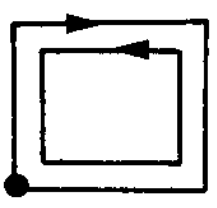

$0(1)$

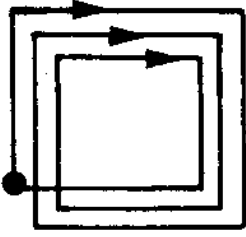

$0(1)$

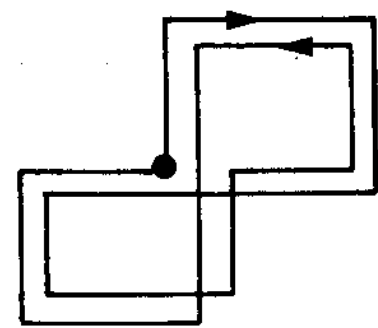

$O(1 / N)$

Fig.1 : Various contributions to $\langle\bar{\psi} \psi\rangle$ at strong coupling : (a) mesonic screening, (b) dynamical fermions, (c) baryonic screening and (d) self-screened meson loop.

of pseudo-Goldstone boson interactions, and one can in fact compare the two sets of results. The regulated lattice theory actually has less number of free parameters and so more predictive power.

- Chiral behaviour is not assumed but is a consequence. No restriction to small quark masses is necessary, and corrections to the leading chiral behaviour can be calculated.

- Unlike the Monte-Carlo calculations, it is easier to work with an infinite lattice than a finite one. This makes it possible to use continuous momenta and directly compute on-shell matrix elements.

The ultimate interest of the lattice game is to extrapolate the results towards the weak coupling limit, and that job still has to be left to Monte-Carlo calculations.

The 4-Fermi operators of the Weak interactions have the structure of a product of two currents. These obey both spinor and colour Fierz identities. The transcription of these operators on the lattice satisfying the spinor Fierz identities involves split-point currents spread over a $2^{4}$ hypercube; and to simultaneously maintain the colour Fierz identities the split vertices of the current are joined by a gauge connection, which after a sum over all equal length paths is projected back on to the $S U(N)$ gauge group [2]. Such a process gives rise to many terms. Only some of them may contribute to a given process as dictated by discrete symmetries, and many of these contributions are equal in the continuum limit.

In the strong coupling limit, I choose to work with correlation functions written in 1 -spinor loop Fierz arrangement and containing 1-link currents. This selection of terms is based on a desire to have correlation functions going over to the correct continuum limit as well as containing only the (partially) conserved vector and axial vector lattice currents. I optimistically hope that the conserved nature of the currents keeps the renormalisation and operator mixing problems under control. To maintain the analogy with the continuum expressions, I use the naive fermion notation :

$$
\begin{aligned}
V_{\mu}^{a}(x) & =\frac{1}{2}\left[\bar{\psi}(x) \gamma_{\mu} U_{\mu}(x) \lambda^{a} \psi(x+\mu)+\bar{\psi}(x+\mu) \gamma_{\mu} U_{\mu}^{\dagger}(x) \lambda^{a} \psi(x)\right] \\
A_{\mu}^{a}(x) & =\frac{1}{2}\left[\bar{\psi}(x) \gamma_{\mu} \gamma_{5} U_{\mu}(x) \lambda^{a} \psi(x+\mu)+\bar{\psi}(x+\mu) \gamma_{\mu} \gamma_{5} U_{\mu}^{\dagger}(x) \lambda^{a} \psi(x)\right]
\end{aligned}
$$

All the correlation functions considered here will be such that they can be spin diagonalised and a factor proportional to the unit matrix can be dropped. The description then changes in to the staggered fermion language. Also it remains transparent that the departure of the lattice correlation functions from the continuum ones is $O\left(a^{2}\right)$, modified by factors of $\ln (a)$ in asymptotically free QCD. 
The strong coupling expansion is an expansion in powers of $1 / N g^{2}$ about the point $g^{2}=\infty$. So it can be thought of as a rearranged $1 / N$ expansion. At $g^{2}=\infty$ the gauge field action is zero, and the correlation functions can be expressed as diagrams containing random walks of colour singlet hadrons. These diagrams can be classified according to the powers of $N$ in their contributions. As a simple illustration diagrams contributing to $\langle\bar{\psi} \psi\rangle$ at different orders in $N$ are shown in Fig.1. It is obvious that terms of arbitrarily high order in $1 / N$ contribute even at $g^{2}=\infty$. I will limit myself to calculating the first two non-trivial terms in such a $1 / N$ series.

The first step in the process is to calculate the lattice meson propagator by resumming a hopping parameter expansion. Then an $n$-point correlation function of quark bilinears can be calculated by adding up all the mesonic Feymman diagrams. It is easier to carry out the calculation in the momentum space, where the constraint of momentum conservation at every vertex is equivalent to summing over all positions of the corresponding vertex in position space. The results for 2 -point functions have been presented in ref.[3]. Using $f_{\pi}$ to fix the lattice scale, the electromagnetic contribution to the pion mass difference was calculated to be, $m_{\pi^{+}}^{2}-m_{\pi^{0}}^{2}=(41.3 \mathrm{MeV})^{2}$, compared with the experimental value of $(35.6 \mathrm{MeV})^{2}$. To keep the expressions for the Weak interaction matrix elements simple, I will here ignore backtracking of random walks and use the large- $d$ limits of the results in ref. $[3]$ :

$$
\begin{gathered}
\kappa=\frac{1}{m+\sqrt{2 d+m^{2}}}, 4 \sinh ^{2}\left(\frac{m_{K}}{2}\right)=\frac{1}{\kappa_{s}}-2 d, \\
f_{K}=\frac{\sqrt{N}}{2}\left(\kappa+\kappa_{s}\right), \Gamma_{0}^{\alpha}(k)=\frac{4 \kappa^{2} \kappa_{s}^{2} \exp \left(i k_{\alpha}\right)}{1-2 \kappa \kappa_{s} \sum_{\mu} \cos k_{\mu}}
\end{gathered}
$$

Here $\kappa$ is the effective quark hopping parameter and $\Gamma_{0}^{\alpha}(k)$ denotes the momentum space $\bar{u} s$-meson propagator with the first and last steps in any and $\alpha^{t h}$ direction respectively.

The expressions in eq.(3) are evaluated at leading order in $N$. The Weak interaction matrix elements are $O\left(N^{2}\right)$ in the same order. They completely factorise and produce results identical to the vacuum insertion values. Up to the same order the Wilson coefficients $C_{i}(\mu)$ are inclependent of the scale $\mu$, and no QCD effects appear.

In order to see interesting effects one has to go to the next order in $N$. Now the QCD effects show up. New operators $O_{i}$ enter the picture, giving rise to correlation functions of $O(N)$. An anomalous dimension matrix appears as well, producing renormalisation group scaling of the Wilson coefficients $C_{i}(\mu)$. In addition to these there are $1 / N$ corrections (e. g., from dynamical fermions) to the leading $O\left(N^{2}\right)$ correlation functions. However, due to the factorisability of the $O\left(N^{2}\right)$ correlation functions, these $1 / N$ effects can be completely absorbed in quantities like $m_{\pi}, f_{\pi}$ and normalisation constants. Therefore, as long as these quantities are factorised out from the final amplitude, it remains consistent to use the expressions in eq.(3) in all correlation functions. In other words, the $1 / N$ corrections to the $O\left(N^{2}\right)$ terms change the physical value of the lattice spacing, but not the dimensionless numbers.

With this machinery in place, it is trivial to calculate the contribution of the $\Delta S=2$ operator to $K^{0}-\bar{K}^{0}$ mixing.

$$
\left\langle K^{-0}\left|(\bar{d} s)_{V-A}(\bar{d} s)_{V-A}\right| \bar{K}^{-0}\right\rangle=2 f_{K^{2}}^{2} M_{K^{-}}^{2}\left(1+\frac{1}{N}\right), \quad M_{K}=2 \sinh \left(\frac{m_{K}}{2}\right)
$$

The 3 -point function involves only a tree graph shown in Fig.2(a). 


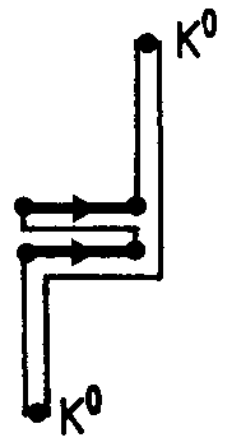

(a)

(a)

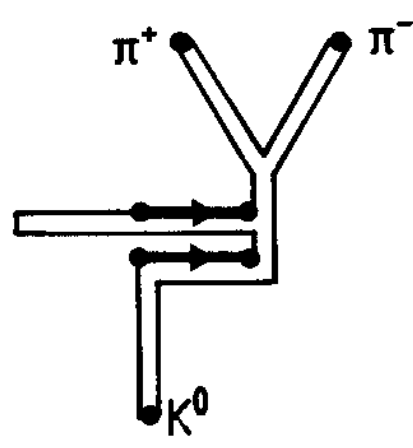

(b)
Fig.2 : (a) A quark level diagram for $K^{0}-\bar{K}^{0}$ mixing, and (b) a diagram for $K^{0} \rightarrow 2 \pi$ decay with a spectator quark.

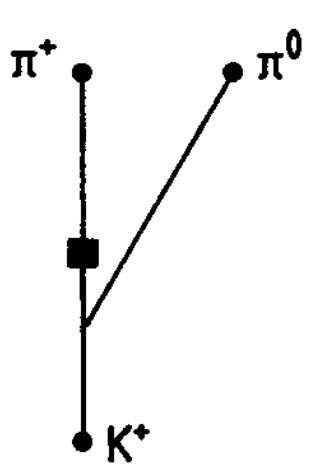

Fig.3: Tree level meson diagrams for $K^{+}$ decay. (a) is $O\left(N^{2}\right)$ and (b) is $O(N)$.

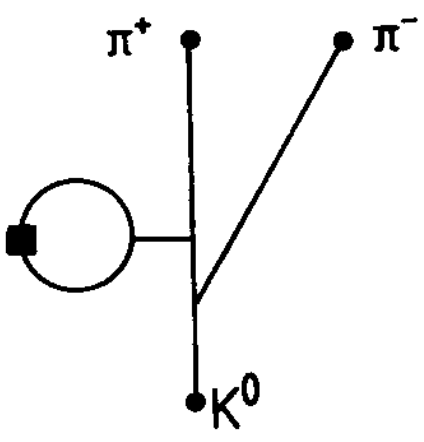

(a)

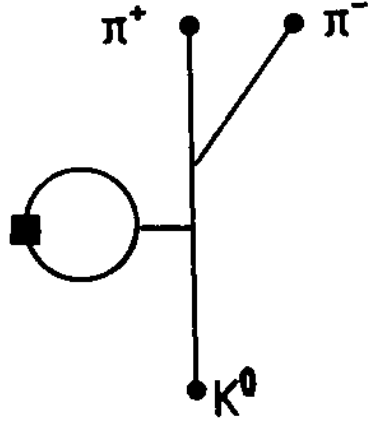

(b)

The $K \rightarrow 2 \pi$ decays require computations of $4-$ point functions. The relevant $4-$ Fermi $\Delta S=1$ operators (without integrating the charm quark out) are :

$O_{ \pm}=\frac{1}{2}\left[(\bar{u} s)_{V-A}(\bar{d} u)_{V-A} \pm(\bar{d} s)_{V-A}(\bar{u} u)_{V-A}-(\bar{c} s)_{V-A}(\bar{d} c)_{V-A} \mp(\bar{d} s)_{V-A}(\bar{c} c)_{V-A}\right]$

The initial condition on the Wilson coefficients at the Weak scale is $C_{+}=C_{-}=1$ [4]. Inclusion of the 1-loop QCD renormalisation effects suppresses $C_{+}$and enhances $C_{-}$[5]. Moreover,

$$
C_{+}+C_{-}=O(1), C_{+}-C_{-}=O\left(\frac{1}{N}\right), C_{-}=\left(C_{+}\right)^{(1+N) /(1-N)} .
$$

First consider the decay $\mathrm{K}^{+} \rightarrow \pi^{+} \pi^{0}$. This $\Delta I=3 / 2$ process again involves only tree level meson diagrams. They are shown in Fig.3(a-b), where the filled and unfilled boxes represent the $4-$ Fermi vertices created by the $W$-boson exchange in the $s$ and $t$ channels respectively. The net result is :

$$
\left\langle K^{+}\left|O_{+}\right| \pi^{+} \pi^{0}\right\rangle=\frac{f_{\pi}}{\sqrt{2}}\left(M_{K}^{2}-M_{\pi}^{2}\right)(1-\delta)\left(1+\frac{1}{N}\right), \delta=\frac{M_{\pi}^{2} \kappa^{2} \kappa_{s}}{\kappa+\kappa_{s}}
$$

The ensuing prediction $\left\langle K^{+}\left|L_{i n t}\right| \pi^{+} \pi^{0}\right\rangle=C_{+}(\mu) \times 0.027 \mathrm{GeV}^{3}$ has to be contrasted with the experimental result $0.010 \mathrm{GeV}^{3}$. 


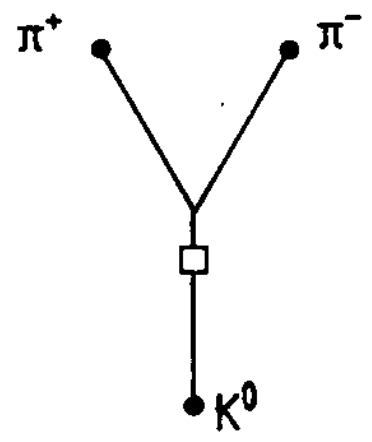

(a)

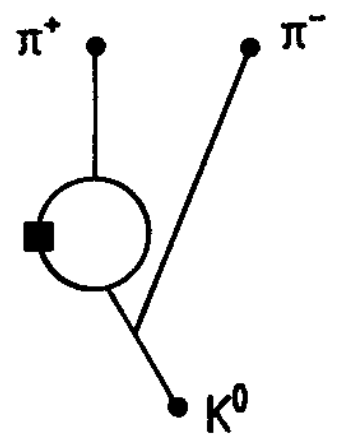

(b)

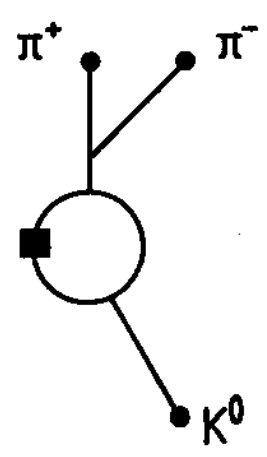

(c)

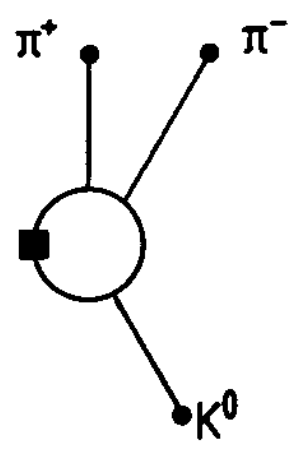

(d)

Fig.5 : Diagrams contributing to $K^{0}$ (but not to $K^{+}$) decay. All are $O(N)$.

The next task is to evaluate the decay amplitude for $K^{0} \rightarrow \pi^{+} \pi^{-}$. The tree diagrams contributing to this process are those of Fig.3(a) (with $K^{+}, \pi^{0}$ replaced by $K^{0}, \pi^{-}$) and Fig.5(a). In addition there are diagrams with an internal meson loop. The diagrams shown in Fig.4(a-b) add up to zero, clearly demonstrating that graphs containing $K^{0} \rightarrow v a c$ as a subprocess cannot contribute to an on-shell decay amplitude. The sum of the diagrams of Fig.5(b-d) however does produce a non-trivial contribution. With the charm quark in the interual loop, and $k_{\mu}$ and $p_{1 \mu}$ respectively denoting the four momenta of the $K^{0}$ and $\pi^{+}$, this contribution is :

$$
\begin{gathered}
E(c)=f_{\pi} \int_{-\pi}^{\pi} \frac{d^{4} l}{(2 \pi)^{4}}\left[\frac{\kappa_{c}^{2}}{\kappa+\kappa_{s}} \cdot \frac{4 A+B \sum_{\mu} \cos l_{\mu}}{\left(1+2 \kappa_{s} \kappa_{c} \sum_{\mu} \cos l_{\mu}\right)\left(1-2 \kappa \kappa_{c} \sum_{\mu} \cos l_{\mu}\right)}\right. \\
\left.\cdot \frac{\kappa_{s} \sum_{\mu} \cos (l-k)_{\mu}-\kappa \sum_{\mu} \cos \left(l-p_{1}\right)_{\mu}}{\left(1+2 \kappa \kappa_{c} \sum_{\mu} \cos \left(l-p_{1}\right)_{\mu}\right)\left(1-2 \kappa \kappa_{c} \sum_{\mu} \cos (l-k)_{\mu}\right)}\right] \\
A=4 \kappa_{s} \kappa_{c}\left(M_{K}^{2}-M_{\pi}^{2}\right), B=4\left(\kappa \kappa_{s}-\kappa_{c}^{2}\right) / \kappa^{2}
\end{gathered}
$$

The integral over the lattice Brillouin zone can be reduced to a three dimensional one using the residue theorem, but then it has to be calculated numerically. I obtain $E(u)-E(c) \sim$ $0.04 f_{\pi}\left(M_{K}^{2}-M_{\pi}^{2}\right)$.

The diagrams for the decay $K^{0} \rightarrow \pi^{0} \pi^{0}$ are topologically the same, but appear with different coefficients. Putting everything together, the full matrix elements become

$$
\begin{array}{r}
\left\langle K^{-0}\left|L_{i n t}\right| \pi^{+} \pi^{-}\right\rangle=\frac{f_{\pi}}{2}\left(M_{K}^{2}-M_{\pi}^{2}\right)\left[C_{-}(\mu)\left(1-\frac{1}{N}\right)\left(1-\delta+\frac{\kappa_{s}}{\kappa}(1+\delta)+0.04\right)\right. \\
\left.+C_{+}(\mu)\left(1+\frac{1}{N}\right)\left(1-\delta-\frac{\kappa_{s}}{\kappa}(1+\delta)-0.04\right)\right] \\
\left\langle K^{-0}\left|L_{i n t}\right| \pi^{0} \pi^{0}\right\rangle=\frac{f_{\pi}}{2 \sqrt{2}}\left(M_{K}^{2}-M_{\pi}^{2}\right)\left[C_{-}(\mu)\left(1-\frac{1}{N}\right)\left(1-\delta+\frac{\kappa_{s}}{\kappa}(1+\delta)+0.04\right)\right. \\
\left.-C_{+}(\mu)\left(1+\frac{1}{N}\right)\left(1-\delta+\frac{\kappa_{s}}{\kappa}(1+\delta)+0.04\right)\right]
\end{array}
$$

These have to be compared with the experimental numbers $0.156 \mathrm{GeV}^{3}$ and $0.105 \mathrm{GeV}^{3}$ respectively. 
The matrix elements in eqs. $(7,10)$ have the correct behaviour in the chiral limit. Also it can be seen that the $K^{+} \rightarrow \pi^{+} \pi^{0}$ and the $K^{-0} \rightarrow \pi^{+} \pi^{-}$decays are $O\left(N^{2}\right)$, but the $K^{-0} \rightarrow \pi^{0} \pi^{0}$ decay is $O(N)$. At this stage there is no real clue as to the appropriate values of the coefficients $C_{ \pm}(\mu)$ at the lattice momentum cut-off scale $\mu=\pi / a$. I can only work backwards and determine that $C_{+}(\mu)=0.33$ and $C_{-}(\mu)=10$ bring the predicted decay rates close to the experimental ones. The justification for such values of the Wilson coefficients at strong coupling is currently under investigation.

The $(V-A)(V+A)$ penguin operators appear in the calculation of $\epsilon^{\prime}$ and in the effective theory where the charm quark is integrated out. Their contribution can be estimated by summing up the diagrams of Fig.5(b-d) with the appropriate spinor structure.

In principle the approach described here can be extended to the Wilson fermion case, with non-perturbative subtractions using operators of dimension $\leq 6$, along the lines of ref.[6]. However, at large values of " $a$ " typical of the strong coupling region, one has to correct for contamination due to operators of dimension $>6$ as well. It is not known whether a consistent description can be found in such a case.

\section{References}

[1] A pedagogical discussion can be found in : H. Georgi, Weak Interactions and Modern Particle Theory (Benjamin/Cummings, 1984).

[2] S. Sharpe, A. Patel, R. Gupta, G. Guralnik and G. Kilcup, Nucl. Phys. B286 (1987) 253.

[3] O. Martin and A. Patel, Phys. Lett. 174B (1986) 94.

[4] A common factor of $G_{F} \sin \theta_{c} \cos \theta_{c} / \sqrt{2}$ has been taken out for brevity.

[5] M. Gaillard and B. Lee, Phys. Rev. Lett. 33 (1974) 108.

G. Altarelli and L. Maiani, Phys. Lett. 52B (1974) 351.

[6] M. Bochicchio, L. Maiani, G. Martinelli, G. C. Rossi and M. Testa, Nucl. Phys. B262 (1985) 331. 